\title{
Intelligent Microcurrent Stimulation System Design
}

\author{
Guomin $\mathrm{Mo}^{1, \mathrm{a}}$, Min Lin ${ }^{2, \mathrm{~b}}$ and Jun-an Zhang ${ }^{2, \mathrm{c}}$ \\ ${ }^{1}$ Science and Technology Department, ${ }^{2}$ Medical Instrumentation College, \\ Shanghai University of Medicine \& Health Sciences, 200093, China \\ aMogm@sumhs.edu.cn, 'binm@sumhs.edu.cn, ' Zjunan@gmail.com
}

Keywords: Intelligent, wireless, CES, EMG, sleep disorder

Abstract. An integrated wireless control and operation, intelligent detection, intelligent control and discharge mode of transcranial electrical stimulation system is designed. The stimulation system can real-time detect and adjust immediately to electromyography, the stimulation parameters according to the results of the test adjust and achieve the best therapeutic effect. Finally, wireless data transmission rate is $20 \mathrm{kbps}$, wireless transceiver frequency is $433 \mathrm{~Hz}$. Adjustable current is $5000 \mu \mathrm{A}$ and adjustable time is 30 minutes.

\section{Introduction}

Cranial electrotherapy stimulation (CES) is a totally different from traditional medication, electric convulsive therapy, non-invasive treatment. CES system can stimulate the brain through constant and low strength Micro current(1-2mA), adjust the cerebral cortex neuron activity, promote the brain secrete a series of neurotransmitters and hormones of diseases such as anxiety, depression, insomnia, closely linked to implement for the treatment of these diseases[1].

CES system can promote to reach the state of "alpha" by changing the abnormal brain waves, promote healthy neurotransmitter secretion, alternative medicine with relaxed and cheerful mood, reduce the stress hormone secretion, improve psychological immunity, which can also quickly change the physiological signals, alleviate pain, improve the physiological signals such as heart rate, blood pressure and muscle tone, effectively control tension anxiety depression and mood state[2][3].

Compared with drug therapy, the treatment does not exist any side effects, and the curative effect is stable, so in Europe, the United States and other countries has been widely used, as a treatment for anxiety, depression, insomnia and related diseases of children a safe and effective treatment.

Existing transcranial electrical stimulator can't to accept transcranial electrical stimulation of the head skin impedance for real-time detection[4], also can't according to the change of impedance and real-time adjustment of voltage and current of constant implementation. If in 15 to 30 minutes of brain stimulation, in the process of conductive adhesive or a thin sponge dipped in salt water in poor contact, loose and conductive performance, will directly affect the effect of electrical stimulation, also unable to detect operators and make corrections in a timely manner.

The key to solve the problem of the technology is to develop a portable wireless control transcranial electrical stimulator which is integrated with the wireless control and operation, intelligent detection, control and discharge model[5]. The stimulator can real-time detection of EMG and adjust the stimulation parameters according to the results of the test, reach to the best treatment effect.

Implantable electrical stimulation system based on wireless communication, according to the requirements of the embedded system technology and characteristics of the embedded system can be divided into the body part and control part in vitro, using wireless communications in vivo and in vitro. Subsystem in the body with the single chip processor as the core, according to the in vitro send command parameters of current wireless controller, and acquisition under the stimulation of EMG feedback to the in vitro. In vitro controller according to the feedback of EMG to adjust the parameter values of the stimulator, in order to achieve better stimulation effect.

\section{Intelligent Microcurrent Stimulation System Structure}

Microcurrent intellectual stimulation and acquisition based on wireless communication system, mainly including electrical stimulation module, myoelectricity acquisition module and 
microprocessor control module, Bluetooth wireless communication module and power module. System architecture is shown in figure 1, in the fission stimulate the collector and the external controller, through wireless module, command parameters and data between internal and external information transmission [6]. Implanted in the body part of the microprocessor as the core, mainly by the electrical stimulation module, myoelectricity acquisition module, power supply module. In vitro control part adopts the same microprocessor as the machine, and by CPU to realize serial communication between upper machine.

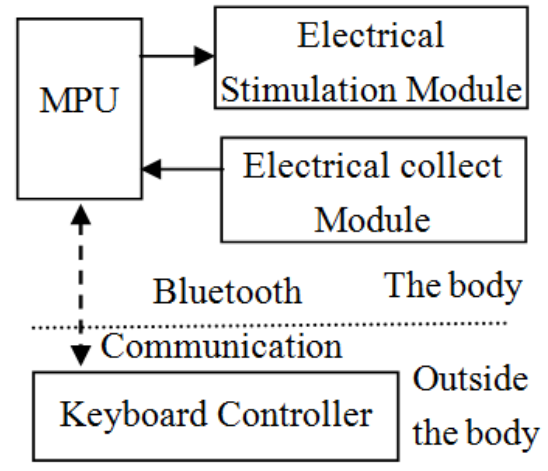

Fig.1. Intelligent Microcurrent stimulation system structure

Figure 2 for the remote control part of the circuit module. In vivo via Bluetooth wireless module and microprocessor control processor communication, receives commands in vitro of the controller, exert the default density and frequency of the micro electric current in the tested muscle tissue; EMG acquisition module real-time acquisition the muscle tissue, muscle electricity information through the microprocessor module converted into the body, then through wireless communication sent to the controller in vitro. Control MCU receives the feedback signal electrode, after scheduled treatment via bluetooth communication terminal to remote control part of the display module. Current sensor selection of hall sensors, electrical testing module includes brain wave sensor, amplifier, filter and AD converter, brain wave sensor by electrode sensor to brain wave signal, after amplification filter, modulus conversion, and sent to MCU control.

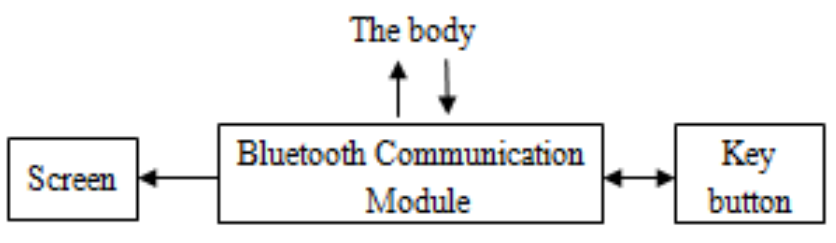

Fig.2. Remote control part of the circuit module

\section{System Working Principle}

Intelligent Microcurrent stimulation system, by the test object implanted wireless module receives commands from in vitro controller, according to the command parameter value SPC two-way required by the stimulus pulse, can response in vitro of ordered at the same time, real-time acquisition of the body muscle electrical signals at the same time, the wireless transmission to in vitro, on the one hand, analysis, application for researchers on the other hand according to the feedback of electromyography signal to adjust the parameter values of the stimulator, in order to achieve better treatment effect.

Firstly, PC will be the default stimulus pulse parameters in the form of the command byte via a serial port to send to the next bit machine, a machine will receive orders from parameters of sorting, packaged in accordance with the requirements of the wireless transport protocol format, and then sent out by in vitro wireless RF module. And wireless module in the body is accepting these commands sent in vitro controller parameters, electrical stimulation by microprocessor guidance module, frequency, pulse width and the intensity of variable polarity stimulation signals, electrical stimulation of nerves and muscles. At the same time, the body's muscle power acquisition module real-time acquisition under the electrical stimulation of EMG, under the control of the microprocessor wireless 
transmission, and the body to the PC via serial communication, PC muscles were collected according to the electric characteristic of the feedback signal to adjust the parameters of the stimulus pulse value, make the system output is more suitable for stimulation waveform, get a better therapeutic effect.

\section{EMG acquisition module}

Microcurrent stimulator excluding signal acquisition module, unable to obtain real-time electrical stimulation effect. This system in the electrical stimulator implanted increased muscle power acquisition module, the module through the sensor acquisition of EMG, after preprocessing the feedback signal to in vitro by wireless transmission mode. Acquisition and processing of EMG as a feedback signal, real-time regulating stimulating pulse parameter values, to achieve a better therapeutic effect[7].

Muscle power acquisition module by a high-pass filter, gain amplification and second-order low-pass filtering units. EMG module circuit diagram is shown in figure 3. Among them, $\mathrm{R} 101=\mathrm{R} 102, \mathrm{C} 101=\mathrm{C} 102$, capacitance of symmetrical two high-pass filter respectively, high-pass filter cut off frequency is $0.08 \mathrm{~Hz}$. CMOS dual op-amp constitute two levels' key low-pass filter, used to remove power frequency interference and noise such as high frequency component. By choosing the appropriate resistance, capacitance, design of low pass filter cutoff frequency is $500 \mathrm{~Hz}$, total amplifier gain reach to 1400 .

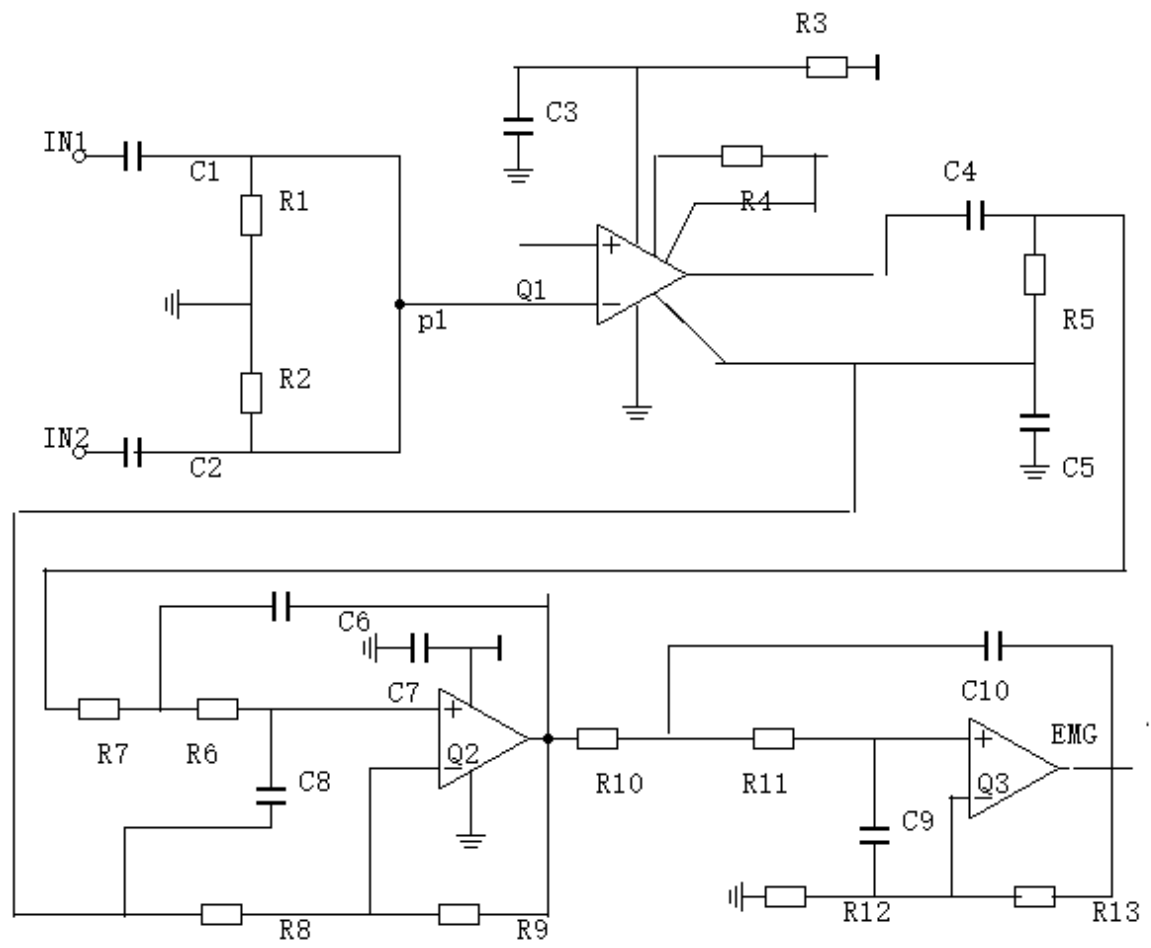

Fig.3. EMG acquisition module

\section{RF wireless communication module design}

For embedded system, wireless communication module choice need to consider the following factors: the number of peripheral components transceiver chip, power consumption and transmission power, etc. Through the analysis of the performance of different chip manufacturer, the system uses the TI company low cost, low energy consumption of the transceiver chip CC1101, realizes the stimulator in vivo and in vitro between wireless communication controllers.

CC1101 through 4 line SPI serial bus interface (SI, SO, the SCLK, CS) connected to the microcontroller P3 port. FIFO (first in first out stacks) data read, write and register configuration. Pin GDO0 and GDO2, other two pin of CC1101, configured to send and receive data packets, connection between the two external interrupt, MCU circuit diagram as shown in figure 4 wireless 
communication module. Data transfer rate of $20 \mathrm{KBPS}$, Wireless transceiver frequency is set at $433 \mathrm{~Hz}$.

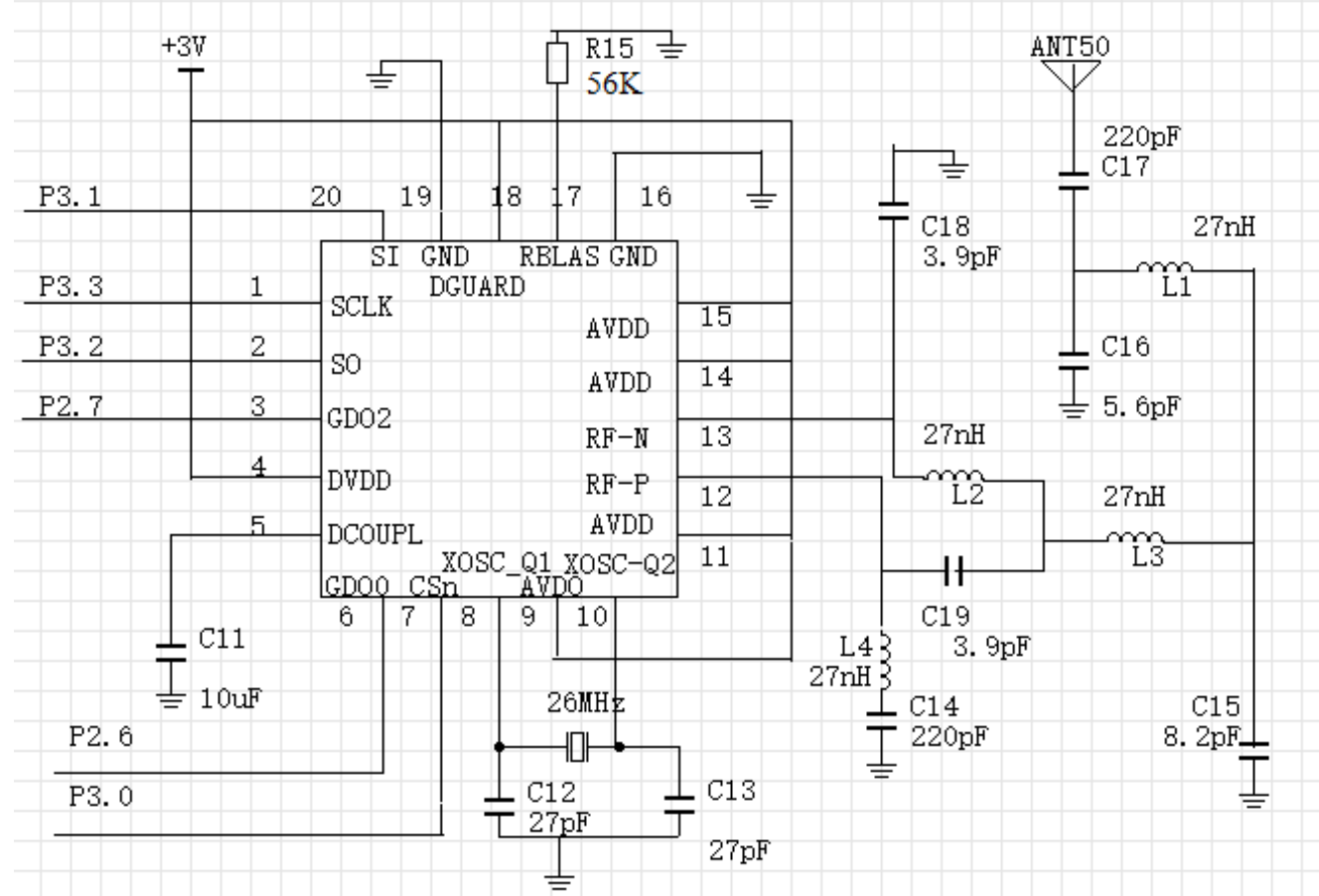

Fig.4. Wireless communication module circuit diagram

RF module wiring, filter capacitance as much as possible close to the device pins to decorate, in order to obtain better filtering effect. As far as possible away from the RF circuit, digital circuit is because there is a steep rise and fall along the digital circuit, can cause interference to radio frequency circuit.

\section{The design of system software}

Software system including system software in the body and in vitro system software two parts.

In vitro system software design. In vitro system via a serial port communication between MCU and PC, a serial port communication protocol: serial communication baud rate to $115200 \mathrm{~b} / \mathrm{s}$. Frame format for one start bit, 8 data bits, no parity, 1 stop bit.

Serial communication data in the form of packet, a set of data at a time. The following PC down a machine to send commands and lower machine to pass data to PC two cases.

PC down a machine to send commands. Data head ( $3 \mathrm{~b})$ parameter 1 : current intensity value $(1 \mathrm{~b})$ parameter 2: pulse width value $(1 \mathrm{~b})$. Parameter 3 : stimulating frequency $(2 \mathrm{~b})$ calibration and $(1 \mathrm{~b})$. Data first $55 \mathrm{~h}$ aaH $00 \mathrm{~h}$ : PC sends a command parameter values to the machine, according to the request of a machine parameter setting corresponding stimulation waveforms, and EMG data. Data first $55 \mathrm{~h}$ aaH $01 \mathrm{~h}$ : PC request a stop of the machine system current stimulation, into the sleep/power saving state; Data at this time the back of the head of the order parameter is empty. Check: for low head start all data from the data accumulation addition eight.

A machine to the PC data transfer. Head (3B) data length (1B) electrical data data (nB) calibration and (1 b) checksum ( 1B ).Data head 55hh: aaH 02H: Show the successful command a machine to receive the upper machine parameters, electrical data will be sent to collect, please PC prepared to receive. The data at the back of the head length value, EMG data is empty. Data head 55 hh: aaH $03 \mathrm{H}$ : Under a machine of electromyography data to PC. At this point the value of the data length is only part of the actual delivery of electrical data bytes, 3 bytes of data does not include the head and one byte of data length. Data head 55hh: aaH 03H: The received command is wrong, request PC resend the order. Check: for low head start all data from the data accumulation addition eight. 


\section{Conclusions}

On the basis of the original system, increased in patients with brain electrical signal acquisition, processing and feedback, and wireless transceiver module, realizes detection, intelligent control and the height of the firing pattern: real-time detection of EMG and adjust the stimulation parameters according to the results of the test, the best treatment effect.

Original system before undergoing brain stimulation, patients need to use eeg instrument analysis of eeg data, according to the analysis result set transcranial electric stimulus, and once set up, in the process of stimulus that cannot be changed. Transcranial electrical stimulation system effectively solved this problem. Transcranial electrical stimulation wireless, intelligent system, can monitor at the time of discharge stimulation in patients with brain electrical response.

In addition, the existing transcranial electrical stimulator use the shielded wire electrodes, electrode film is more than the use of conductive adhesive or a thin sponge dipped in salt water as a medium of contact with the scalp, the operator according to the need to stimulate the brain is stimulated in the man's head corresponding to find the location, and then respectively on different electrodes with elastic tape head fixed contact. As a result, the whole process of transcranial electrical stimulation, and subject to the length of the wire, the size of the electrodes, and fixed tape firmly, accept the electrical stimulation of the brain were often asked to not free to move in the process of stimulating, or be restricted to some head movements.

By patients (sleep) in patients with analysis of EEG signals, real-time tracking, feedback and handling system, according to different patients intelligence analysis, judgment and processing, automatic stimulation parameters adjusting and optimizing the setting, the best treatment effect. The wireless transceiver module makes the system easy to operate, more practical and durable, easy to promote.

EMG acquisition module to realize the collection of the EMG. CMOS dual op-amp circuit composition, two levels of 'base, low-pass filter, eliminates the power frequency interference and high frequency component.

RF modules realize wireless communication signal. Wireless data transfer rate of 20Kbps; Wireless transceiver set $433 \mathrm{~Hz}$ frequency. System software including in vivo and in vitro controller software, they cooperate to achieve the precision Microcurrent stimulation and intelligent control system.Product use micro power wireless ISM frequency band transmission (0DBMW), power and power density is far lower than the medical equipment EMC standard requirements.

\section{Acknowledgements}

This work was financially supported by Shanghai Science and Technology Commission Research Project Foundations, Cooperation projects by the department of Production, Schools, Research, Development and Medical (12DZ1940904).

\section{References}

[1] Hummel F, Celnik P, Giraux Petal. Brain( 2005)

[2] Baker J M, Rorden C, Fridriksson J. Stroke(2010)

[3] Zhaoyuan Wang and Longqi Zhou. J First MII Med. Univ. 20(2), 189-190 (2000),In Chinese

[4] G.M. Mo, Y.H. Guo, S. M. Mao and J. A. Zhang, Applied Mechanics and Materials, v 631-632, pp.387-394( 2014)

[5] Weiming Wang, Bozhi Ma and Hongwei Hao. Chinese Journal of Biomedical Engineering, 28(3):408-414(2009). in Chinese

[6] Min Lin, Yanlin Shan, Guomin Mo. Chinese Patent:2012 2 0371840. 5(2012).In Chinese

[7] Min Lin, Guomin Mo. Beijing Biomedical Engineering. Vol.32. No.1:72-77 (2013). In Chinese 